\title{
NDF of the near-field of a strip source in orthogonal directions
}

\author{
Rocco Pierri, Raffaele Moretta, Student Member, IEEE
}

\begin{abstract}
In this paper, we address the problem of computing the NDF (number of degrees of freedom) of the field radiated by a strip source along all the possible directions orthogonal to it. As well known, the $N D F$ is equal to the number of singular values of the radiation operator that are before a critical index at which they abrupt decay. Unfortunately, in the considered case the solution of the associate eigenvalue problem is not known in closed-form, and this prevents us from directly evaluating the singular values of the radiation operator.

To overcome this drawback, in this paper we exploit a weighted adjoint operator. The latter allows obtaining an eigenvalue problem whose solution is known in closed-form but, at the same time, it changes the behavior of the singular values. However, since the modification of the singular values involves only the dynamics but not the critical index at which they abrupt decay, we succeed in the evaluation of the NDF of the radiated field.
\end{abstract}

Index Terms-inverse source problem, radiation operator, integral equation, eigenvalue problem, singular values, $N D F$.

\section{INTRODUCTION}

The inverse source problem has several applications in antenna analysis and synthesis [1]-[9]. From the mathematical point of view, it entails inverting a linear integral operator, called radiation operator, which links the density current $J$ to the radiated field $E$. The latter represent respectively the unknown and the data of the inverse problem.

In order to evaluate the achievable performance in linear inverse problems, some common metrics are the point spread function (PSF) [10], and the number of degrees of freedom $(N D F)$ [11]-[12]. In particular, the point spread function provides the reconstruction of an impulsive source or the synthesis of an impulsive field; instead, the number of degrees of freedom represents at the same time the number of independent functions required to represent the data with a given degree of accuracy, and the dimension of the unknowns subspace that can be stably reconstructed.

Both the metrics depend on the geometric parameters of the configuration and both are linked to the singular values decomposition $(S V D)$ of the radiation operator, which is a key mathematical tool widely used in electromagnetics [13][17]. The link between the singular values decomposition and the metrics mentioned above appears immediately clear if we consider that the point spread function can be expressed in terms of the singular system of the radiation operator while

Manuscript received November 19, 2020.

This work has been supported by the European Union and the Italian Ministry of University and Research funding through Programma Operativo Nazionale Ricerca e Innovazione 2019/2020 under Grant B26C18000080005. (Corresponding author: Raffaele Moretta.)

The authors are with the Department of Engineering, University of Campania "Luigi Vanvitelli", 81031 Aversa, Italy (email: raffaele.moretta@unicampania.it). the number of degrees of freedom is given by the number of relevant singular values of such an operator [18] [19]. However, the $S V D$ of the radiation operator can be exploited not only to evaluate the singular system, the number of degrees of freedom, and the point spread function [20]-[23] but also to find a discretization scheme of the radiation operator that exploits a non-redundant number of samples [24]-[27].

In this article, we refer to the configuration consisting of a magnetic current strip observed on a truncated line orthogonal to the source. Such geometry has been considered in [28] [29] for the study of the depth resolution only in the case where the observation domain is centered with respect to the source. Differently, here we focus on the prevision of the $N D F$ by removing the symmetry condition on the observation domain.

Let us remark that the study of the NDF for the configuration described before not only gives insight both on the forward and on inverse source problems but it is of great interest also in phase retrieval when the phaseless data are collected on more scanning surfaces parallel to each other. In the latter context, the knowledge of the $N D F$ along the orthogonal directions allows establishing the number of scanning surfaces which provides independent information. In fact, taking into account that the square amplitude of the radiated field must be sampled with a step-length that is the half of the radiated field, it is possible to retain that the number of scanning surface which allows collecting independent data will be approximately equal to two times the $N D F$ of the radiated field.

For the considered configuration, since the kernel of the related eigenvalue problem is space-variant, the issue of finding the $N D F$ by studying the singular values behavior of the radiation operator is quite difficult. To surmount this drawback, first we introduce a weighted adjoint and we explicit the kernel of the eigenvalue problem by exploiting asymptotic arguments. After, by performing a change of variables, we recast the original eigenvalue problem in a new one.

If the observation domain is outside the source domain or centered with respect to it, the new eigenvalue problem involves a convolution operator with a sinc kernel. Hence, its eigenvalues can be computed by exploiting the closedform results related to the Slepian-Pollak operator [30]-[31]. At this stage, the $N D F$ of the radiated field will be obtained by observing that the presence of the weight function in the adjoint operator affects only the dynamics of the eigenvalues but it does not change the position of the knee; hence, the $N D F$ corresponds the number of relevant eigenvalues of the sinc kernel.

Differently, if the observation domain is in front of the source but not centered with respect to it, the kernel of the 
new eigenvalue problem can be expressed by a sinc function of difference type plus an error term that does not affect the number of relevant eigenvalues. As we will show, also in this case the presence of the weight function does not affect the position of the knee in the eigenvalues diagram. Hence, the $N D F$ of the radiated field can be still evaluated by referring to the integral operator with the sinc kernel. Consequently, our final result will be that of providing the $N D F$ of the field for each possible observation domain orthogonal to the source.

The article is organized as follows. In section II we show the geometry of the problem, and we introduce the radiation operator and its adjoint. In section III, we introduce the kernel involved in the integral equation for the computation of the singular values of the radiation operator. In section IV and V, first we evaluate such kernel in the case where the observation domain is located respectively in the region outside the source, and in the region in front of the source. After that, we show how it is possible to recast such kernel in a form more similar to a sinc kernel of difference type. In section VI, we find the number of degrees of freedom of the radiated field. A section of conclusion follows.

\section{GEOMETRY OF THE PROBLEM}

A magnetic current $J(x)=J(x) \hat{i}_{y}$, supported on the set $S D=[-a, a]$ of the $x$-axis, radiates in a homogeneous medium with wavenumber $\beta$. The electric field radiated by such current has two components one along the $x$ axis, and another along the $z$-axis. The $x$ component of the electric field, $E$, is observed on a bounded observation domain $O D=$ $\left[z_{\min }, z_{\max }\right]$ that is orthogonal to the source, and located in near non-reactive along the axis $x=x_{o}$.

For the considered configuration, the radiation operator $\mathcal{T}$ is given by

$$
\mathcal{T} J=\int_{-a}^{a} z \frac{e^{-j \beta R\left(x^{\prime}, z\right)}}{R^{\frac{3}{2}}\left(x^{\prime}, z\right)} J\left(x^{\prime}\right) d x^{\prime}
$$

where $R\left(x^{\prime}, z\right)=\sqrt{\left(x_{o}-x^{\prime}\right)^{2}+z^{2}}$.

The adjoint operator $\mathcal{T}^{\dagger}$ is usually defined as below

$$
<\mathcal{T} J, E>_{L^{2}\left[z_{\min }, z_{\max }\right]}=\left\langle J, \mathcal{T}^{\dagger} E\right\rangle_{L^{2}[-a, a]}
$$

with $<,>_{L^{2}}$ denoting the scalar product in the functional space $L^{2}$ [32]. Here, we do not refer to the usual adjoint operator defined in (2) but we introduce a weighted adjoint operator given by

$$
\mathcal{T}_{w}^{\dagger} E=\mathcal{T}^{\dagger} w E=\int_{z_{\min }}^{z_{\max }} w\left(x^{\prime}, z\right) z \frac{e^{j \beta R\left(x^{\prime}, z\right)}}{R^{\frac{3}{2}}\left(x^{\prime}, z\right)} E(z) d z
$$

where $w\left(x^{\prime}, z\right)$ denotes a real positive continuous function that pre-weights / pre-filters the data.

As will be more clear in next sections, the use of the weighted adjoint has two effects. On one hand, it simplifies the mathematic analysis of the radiation operator. On the other, it changes the singular values decomposition of the radiation operator. Naturally, the latter would appear a bad effect. However, as we will show, the presence of the weight function changes only the shape of the singular values but not the critical index at which they abrupt decay; hence, we can

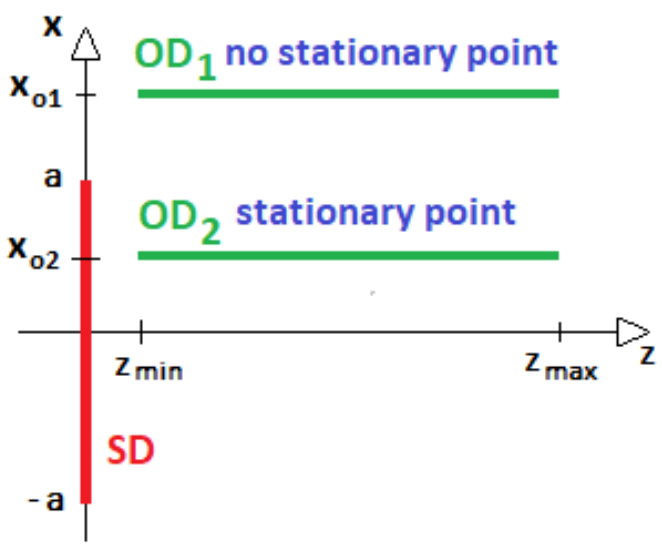

Fig. 1. Geometry of the problem

use the weight adjoint for evaluating the $N D F$ of the radiated field.

\section{Preliminaries Concepts for the StUdy OF THE RADIATION OPERATOR}

With the aim to study the singular values behavior of the radiation operator $\mathcal{T}$, we will study the eigenvalues of the auxiliary operator $\mathcal{T} \mathcal{T}_{w}^{\dagger}$. By virtue of the definitions (1) and (3), the latter can be expressed as below

$$
\mathcal{T} \mathcal{T}_{w}^{\dagger} E=\int_{z_{\min }}^{z_{\max }} K\left(z_{o}, z\right) E(z) d z
$$

where

$$
K\left(z_{o}, z\right)=z_{o} z \int_{-a}^{a} w\left(x^{\prime}, z\right) \frac{e^{-j \beta\left[R\left(x^{\prime}, z_{o}\right)-R\left(x^{\prime}, z\right)\right]}}{R^{\frac{3}{2}}\left(x^{\prime}, z_{o}\right) R^{\frac{3}{2}}\left(x^{\prime}, z\right)} d x^{\prime}
$$

By setting $f\left(x^{\prime}, z_{o}, z\right)=1 /\left[R^{\frac{3}{2}}\left(x^{\prime}, z_{o}\right) R^{\frac{3}{2}}\left(x^{\prime}, z\right)\right]$ and $\phi\left(x^{\prime}, z_{o}, z\right)=\left[R\left(x^{\prime}, z_{o}\right)-R\left(x^{\prime}, z\right)\right] / a$, the kernel can be recast in the following form

$K\left(z_{o}, z\right)=z_{o} z \int_{-a}^{a} w\left(x^{\prime}, z\right) f\left(x^{\prime}, z_{o}, z\right) e^{-j \beta a \phi\left(x^{\prime}, z_{o}, z\right)} d x^{\prime}$

For $z_{o}=z$ the exponential function in (6) is equal to one; consequently, the kernel $K\left(z_{o}, z\right)$ can be exactly computed by exploiting the integration by part method.

For each $\left(z_{o}, z\right) \in\left[z_{\min }, z_{\max }\right] \times\left[z_{\min }, z_{\max }\right]:\left\{z_{o} \neq z\right\}$, if $\beta a>>1$, the integral above can be evaluated by resorting to an asymptotic approach. Since the choice of the asymptotic technique is related to the presence/absence of stationary points, it arises the problem of solving the equation

$$
\phi^{\prime}\left(x^{\prime}, z_{o}, z\right)=0 \quad \forall x^{\prime} \in[-a, a]
$$

where $\phi^{\prime}$ indicates the partial derivative of $\phi$ with respect to $x^{\prime}$. It is easy to show that if $\left|x_{o}\right|>a$ (or in other words if the observation domain is outside the region of the source), equation (7) does not admit solution any solution with respect to $x^{\prime}$ in the set $x^{\prime} \in[-a, a]$. Differently, if $\left|x_{o}\right|<a$ (or equivalently if the observation domain is located in front of the source), the point $x^{\prime}=x_{o}$ is a solution of such equation (see fig. 1). 


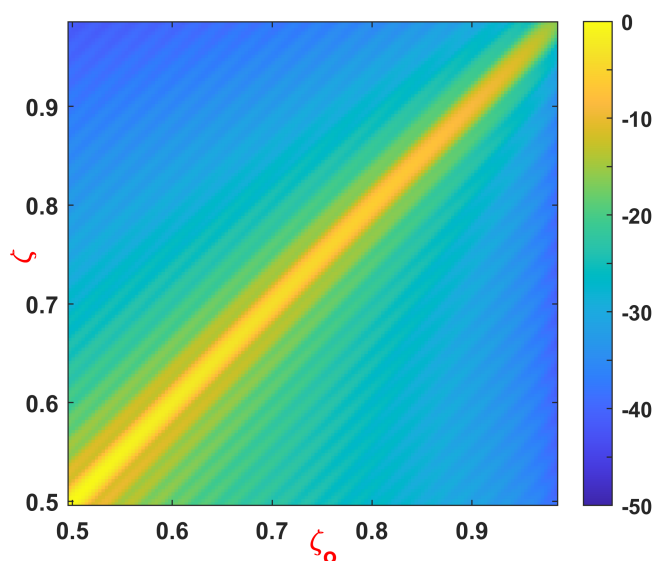

Fig. 2. Kernel of $\mathcal{T} \mathcal{T}^{\dagger}$ in the variables $\left(\zeta, \zeta_{o}\right)$ for $a=20 \lambda, x_{o}=25 \lambda$, $z_{\min }=2.5 \lambda\left(\zeta_{\max }=0.987\right), z_{\max }=40 \lambda\left(\zeta_{\min }=0.497\right)$.

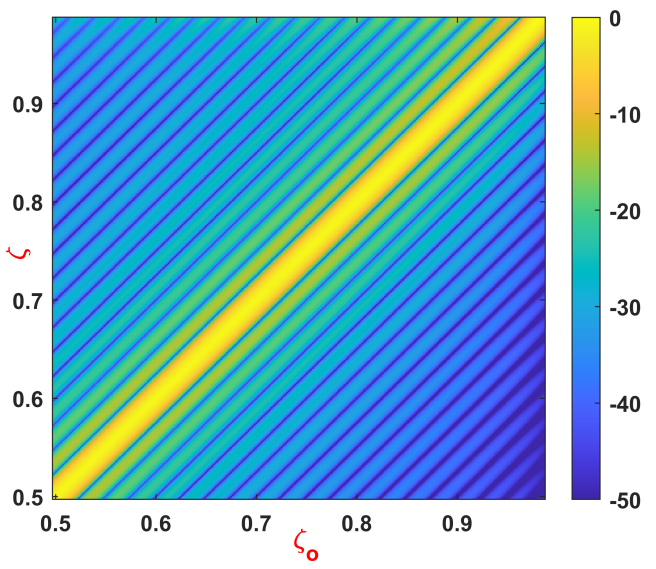

Fig. 3. Kernel of $\mathcal{T} \mathcal{T}_{w}^{\dagger}$ in the variables $\left(\zeta_{o}, \zeta\right)$ for $a=20 \lambda, x_{o}=25 \lambda$, $z_{\min }=2.5 \lambda\left(\zeta_{\max }=0.987\right), z_{\max }=40 \lambda\left(\zeta_{\min }=0.497\right)$.

For such reason, the cases $\left|x_{o}\right|>a$ and $\left|x_{o}\right|<a$ will be analyzed separately in sections IV and V, respectively. Furthermore, it is worth noting that the study of $\mathcal{T} \mathcal{T}_{w}^{\dagger}$ for $x=-x_{o}$ can be done in dual way with respect to the case $x=x_{o}$. This allows us to address only the cases where $x_{o} \geq 0$.

\section{STUdY OF $\mathcal{T} \mathcal{T}_{w}^{\dagger}$ IN THE CASE $x_{o}>a$}

In this section, we first evaluate the kernel of the operator $\mathcal{T} \mathcal{T}_{w}^{\dagger}$ in the case where $x_{o}>a$. Later, we show how to recast such operator in a form more similar to a convolution operator with a bandlimited kernel of sinc type.

As seen before, for $x_{o}>a$ no stationary points appear in the phase function; hence, the integral (6) can be asymptotically evaluated by taking into account only the contributions of the endpoints [33]. Consequently, if $\beta a>>1$ and $x_{o}>a$ then $\forall\left(z_{o}, z\right) \in\left[z_{\min }, z_{\max }\right] \times\left[z_{\min }, z_{\max }\right]:\left\{z_{o} \neq z\right\}$ the kernel of $\mathcal{T} \mathcal{T}_{w}^{\dagger}$ can be approximated by

$$
\begin{aligned}
& K\left(z_{o}, z\right) \approx \\
& -\left.\frac{z_{o} z}{j \beta a}\left(w\left(x^{\prime}, z\right) \frac{f\left(x^{\prime}, z_{o}, z\right)}{\phi^{\prime}\left(x^{\prime}, z_{o}, z\right)} e^{-j \beta a \phi\left(x^{\prime}, z_{o}, z\right)}\right)\right|_{x^{\prime}=-a} ^{x^{\prime}=a}
\end{aligned}
$$

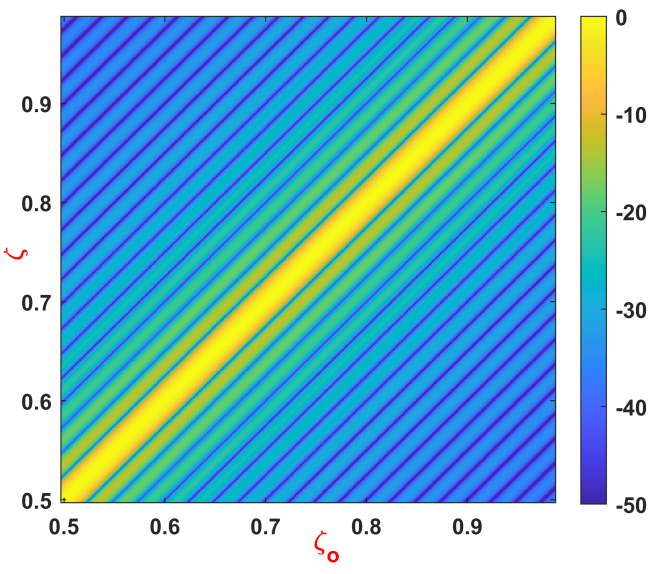

Fig. 4. Sinc kernel given by (17) for $a=20 \lambda, x_{o}=25 \lambda, z_{\min }=2.5 \lambda$ $\left(\zeta_{\max }=0.987\right), z_{\max }=40 \lambda\left(\zeta_{\min }=0.497\right)$.

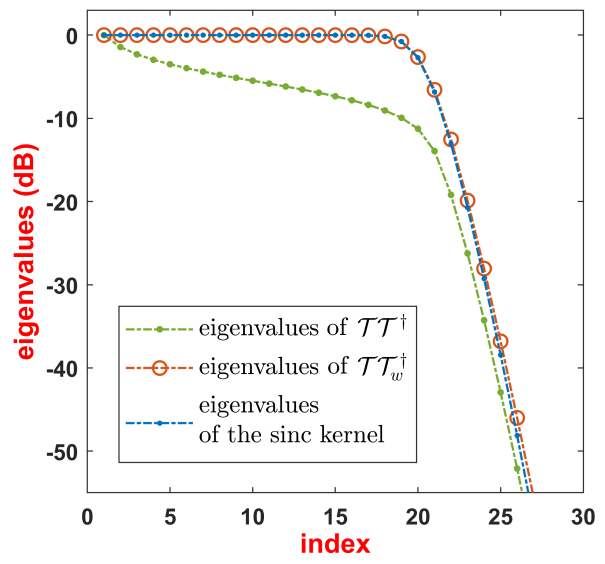

Fig. 5. Eigenvalues of $\mathcal{T} \mathcal{T}^{\dagger}, \mathcal{T} \mathcal{T}_{w}^{\dagger}$, and of the sinc kernel for $a=20 \lambda$, $x_{o}=25 \lambda, z_{\min }=2.5 \lambda\left(\zeta_{\max }=0.987\right), z_{\max }=40 \lambda\left(\zeta_{\min }=0.497\right)$.

From equation (8), it follows that

$$
\begin{aligned}
& K\left(z_{o}, z\right) \approx-\frac{z_{o} z}{j \beta a} e^{-j \frac{\beta a}{2}\left(\phi_{a}+\phi_{-a}\right)} . \\
& \left(\frac{w_{a} f_{a}}{\phi_{a}{ }^{\prime}} e^{j \frac{\beta a}{2}\left(\phi_{-a}-\phi_{a}\right)}-\frac{w_{-a} f_{-a}}{\phi_{-a}{ }^{\prime}} e^{-j \frac{\beta a}{2}\left(\phi_{-a}-\phi_{a}\right)}\right)
\end{aligned}
$$

where the subscripts $-a$ or $a$ indicate that the correspondent function has been particularized in $x^{\prime}=-a$ or $x^{\prime}=a$.

As can be seen from (9), the operator $\mathcal{T} \mathcal{T}_{w}^{\dagger}$ is space-variant. In order to recast it in a form more similar to a convolution operator, let us introduce the following variables

$$
\begin{aligned}
& \zeta(z)=\frac{1}{2 a}\left(\sqrt{\left(x_{o}+a\right)^{2}+z^{2}}-\sqrt{\left(x_{o}-a\right)^{2}+z^{2}}\right) \\
& \gamma(z)=\frac{1}{2 a}\left(\sqrt{\left(x_{o}+a\right)^{2}+z^{2}}+\sqrt{\left(x_{o}-a\right)^{2}+z^{2}}\right)
\end{aligned}
$$

which represent the two elliptic coordinates. Thanks to the introduction of such variables, it results that $\frac{\beta a}{2}\left(\phi_{-a}-\phi_{a}\right)=$ $\beta a\left(\zeta_{o}-\zeta\right)$ and $\frac{\beta a}{2}\left(\phi_{-a}+\phi_{a}\right)=\beta a\left(\gamma\left(\zeta_{o}\right)-\gamma(\zeta)\right)$. Hence, by passing from the variables $\left(z_{o}, z\right)$ to the variables $\zeta_{o}=\zeta\left(z_{o}\right)$ and $\zeta=\zeta(z)$, it is possible to recast (4) as below

$$
\mathcal{T} \mathcal{T}_{w}^{\dagger} E=\int_{\zeta\left(z_{\max }\right)}^{\zeta\left(z_{\min }\right)} K\left(\zeta_{o}, \zeta\right) E(\zeta) d \zeta
$$


where

$$
\begin{gathered}
K\left(\zeta_{o}, \zeta\right) \approx \frac{z\left(\zeta_{o}\right) z(\zeta)}{j \beta a} \frac{d z}{d \zeta} e^{-j \beta a\left[\gamma\left(\zeta_{o}\right)-\gamma(\zeta)\right]} \\
\left(\frac{w_{a}(\zeta) f_{a}\left(\zeta_{o}, \zeta\right)}{\phi_{a}{ }^{\prime}\left(\zeta_{o}, \zeta\right)} e^{j \beta a\left(\zeta_{o}-\zeta\right)}-\frac{w_{-a}(\zeta) f_{-a}\left(\zeta_{o}, \zeta\right)}{\phi_{-a}{ }^{\prime}\left(\zeta_{o}, \zeta\right)} e^{-j \beta a\left(\zeta_{o}-\zeta\right)}\right) .
\end{gathered}
$$

The full expression of $z(\zeta)$ and its derivative $\frac{d z}{d \zeta}$ is reported in appendix A.

At this stage, the kernel has still a complicate structure. However, by approximating the amplitude factors as follows

$$
\begin{gathered}
\frac{z\left(\zeta_{o}\right) f_{a}\left(\zeta_{o}, \zeta\right)}{\phi_{a}^{\prime}\left(\zeta_{o}, \zeta\right)} \approx \frac{a}{\left(x_{o}-a\right)\left(\zeta_{o}-\zeta\right) \frac{d z}{d \zeta}} \\
\frac{z\left(\zeta_{o}\right) f_{-a}\left(\zeta_{o}, \zeta\right)}{\phi_{-a}^{\prime}\left(\zeta_{o}, \zeta\right)} \approx \frac{a}{\left(x_{o}+a\right)\left(\zeta_{o}-\zeta\right) \frac{d z}{d \zeta}}
\end{gathered}
$$

and by choosing

$$
w\left(x^{\prime}, \zeta\right)=\frac{\bar{w}\left(x^{\prime}\right)}{z(\zeta)}=\frac{\left(x_{o}-x^{\prime}\right)}{z(\zeta)},
$$

the kernel can be rewritten in simple and nice form

$$
K\left(\zeta_{o}, \zeta\right) \approx \frac{2 \pi}{\beta} e^{j \beta a\left[\gamma(\zeta)-\gamma\left(\zeta_{o}\right)\right]} \frac{\sin \left(\beta a\left(\zeta_{o}-\zeta\right)\right)}{\pi\left(\zeta_{o}-\zeta\right)} .
$$

The derivation of the approximations (14) and (15) is shown in Appendix B.

Figures 2, 3 and 4 sketch respectively the kernel of $\mathcal{T T}^{\dagger}$, $\mathcal{T} \mathcal{T}_{w}^{\dagger}$, and the sinc kernel (17) in $d B$. As can be seen from the figures, the sinc kernel is almost equal to the actual kernel of $\mathcal{T} \mathcal{T}_{w}^{\dagger}$. Consequently, the eigenvalues of the sinc kernel (17) approximate very well those of $\mathcal{T} \mathcal{T}_{w}^{\dagger}$. This is confirmed also by fig. 5 in which the eigenvalues of $\mathcal{T} \mathcal{T}^{\dagger}$, those of $\mathcal{T} \mathcal{T}_{w}^{\dagger}$, and those of the sinc kernel (17) are depicted.

As can be seen from such figure, the eigenvalues of $\mathcal{T} \mathcal{T}_{w}^{\dagger}$ and those of the sinc kernel (17) have the same behavior, instead, the eigenvalues of $\mathcal{T} \mathcal{T}_{w}^{\dagger}$ exhibit a different behavior with respect to those of $\mathcal{T} \mathcal{T}^{\dagger}$. Despite this, the critical index beyond which the eigenvalues abrupt decay is the same for both the operators. This means that the use of the weight adjoint does not change the number of relevant singular values but it modifies only the dynamics of the singular values by making them more flat.

From the considerations above, it follows that the number of relevant eigenvalues of $\mathcal{T T}^{\dagger}$ (or in the words the NDF of the radiated field) can be evaluated by determining the number of relevant eigenvalues of $\mathcal{T} \mathcal{T}_{w}^{\dagger}$ which, in turn, exhibits the same eigenvalues of the integral operator with the sinc kernel (17).

\section{STUdy OF $\mathcal{T} \mathcal{T}_{w}^{\dagger}$ IN THE CASE $0 \leq x_{o} \leq a$}

In this section, with reference to the case where $0 \leq x_{o} \leq a$, we will evaluate the kernel of $\mathcal{T} \mathcal{T}_{w}^{\dagger}$ and we will recast it in a form suitable to be studied.

Differently from the case in section IV, if $0<x_{o}<a$ the asymptotic evaluation of the kernel contains not only the contributions of the endpoints $x^{\prime}=-a$ and $x^{\prime}=a$ but also the contribution given by the stationary point $x^{\prime}=x_{o}$ [33]. Hence, if $\beta a>>1$ and $0<x_{o}<a$ an asymptotic evaluation of the integral (6) which holds true $\forall\left(z_{o}, z\right) \in$ $\left[z_{\min }, z_{\max }\right] \times\left[z_{\min }, z_{\max }\right]:\left\{z_{o} \neq z\right\}$ is given by

$$
\begin{aligned}
K\left(z_{o}, z\right) \approx z_{o} z & \left(\frac{w_{a} f_{a}}{-j \beta a \phi_{a}^{\prime}} e^{-j \beta a \phi_{a}}-\frac{w_{-a} f_{-a}}{-j \beta a \phi_{-a}{ }^{\prime}} e^{-j \beta a \phi_{-a}}\right. \\
+ & \left.\sqrt{\frac{2 \pi}{\beta a}} \frac{w_{x_{o}} f_{x_{o}}}{\sqrt{\left|\phi_{x_{o}}^{\prime \prime}\right|}} e^{-j \beta a \phi_{x_{o}}} e^{j \frac{\pi}{4} \operatorname{sign}\left(-\phi_{x_{o}}^{\prime \prime}\right)}\right)
\end{aligned}
$$

where

- $\phi^{\prime \prime}$ indicates the second partial derivative of $\phi$ with respect to the variable $x^{\prime}$,

- the subscripts $-a, a, x_{o}$ denote respectively that the corresponding function has been particularized for $x^{\prime}=-a$, $x^{\prime}=a$ or $x^{\prime}=x_{o}$.

The previous expression of $K\left(z_{o}, z\right)$ can be easily recast in the form

$$
\begin{aligned}
& K\left(z_{o}, z\right) \approx z_{o} z e^{-j \frac{\beta a}{2}\left(\phi_{-a}+\phi_{x_{o}}\right)}\left(\frac{w_{-a} f_{-a}}{j \beta a \phi_{-a}{ }^{\prime}} e^{-j \frac{\beta a}{2}\left(\phi_{-a}-\phi_{x_{o}}\right)}\right. \\
& +\sqrt{\frac{2 \pi}{\beta a}} \frac{w_{x_{o}} f_{x_{o}}}{\sqrt{\left|\phi_{x_{o}}^{\prime \prime}\right|}} e^{j \frac{\beta a}{2}\left(\phi_{-a}-\phi_{x_{o}}\right)} e^{j \frac{\pi}{4} \operatorname{sign}\left(z_{o}-z\right)}-\Delta K\left(z_{o}, z\right)
\end{aligned}
$$

where

$$
\Delta K\left(z_{o}, z\right)=\frac{z_{o} z}{j \beta a} \frac{w_{a}(z) f_{a}\left(z_{o}, z\right)}{\phi_{a}{ }^{\prime}\left(z_{o}, z\right)} e^{-j \beta a \phi_{a}\left(z_{o}, z\right)} .
$$

With the aim to obtain a kernel whose eigenvalues can be computed in closed form, let us introduce the variables

$$
\begin{aligned}
& \zeta(z)=\frac{1}{a+x_{o}}\left(\sqrt{\left(x_{o}+a\right)^{2}+z^{2}}-|z|\right) \\
& \gamma(z)=\frac{1}{a+x_{o}}\left(\sqrt{\left(x_{o}+a\right)^{2}+z^{2}}+|z|\right)
\end{aligned}
$$

The latter allow writing that $a\left(\phi_{-a}-\phi_{x_{o}}\right)=\left(a+x_{o}\right)\left(\zeta_{o}-\zeta\right)$ and $a\left(\phi_{-a}\left(\zeta_{o}, \zeta\right)+\phi_{x_{o}}\left(\zeta_{o}, \zeta\right)\right)=\left(a+x_{o}\right)\left(\gamma\left(\zeta_{o}\right)-\gamma(\zeta)\right)$. Hence, by expressing (3) in terms of the variables $\zeta_{o}=\zeta\left(z_{o}\right)$ and $\zeta=\zeta(z)$, we have that

$$
\mathcal{T} \mathcal{T}_{w}^{\dagger} E=\int_{\zeta\left(z_{\max }\right)}^{\zeta\left(z_{\min }\right)} K\left(\zeta_{o}, \zeta\right) E(\zeta) d \zeta
$$

where

$$
\begin{aligned}
& K\left(\zeta_{o}, \zeta\right) \approx-z\left(\zeta_{o}\right) z(\zeta) \frac{d z}{d \zeta} e^{-j \frac{\beta\left(a+x_{o}\right)}{2}\left(\gamma\left(\zeta_{o}\right)-\gamma(\zeta)\right)} \\
& \left(w_{-a}(\zeta) \frac{f_{-a}\left(\zeta_{o}, \zeta\right)}{j \beta a \phi_{-a}^{\prime}\left(\zeta_{o}, \zeta\right)} e^{-j \frac{\beta\left(a+x_{o}\right)}{2}\left(\zeta_{o}-\zeta\right)}+\sqrt{\frac{2 \pi}{\beta a}} w_{x_{o}}(\zeta)\right. \\
& \left.\frac{f_{x_{o}}\left(\zeta_{o}, \zeta\right)}{\sqrt{\left|\phi_{x_{o}}^{\prime \prime}\left(\zeta_{o}, \zeta\right)\right|}} e^{\frac{\beta\left(a+x_{o}\right)}{2}\left(\zeta_{o}-\zeta\right)} e^{-j \frac{\pi}{4} \operatorname{sign}\left(\zeta_{o}-\zeta\right)}\right)+\Delta K\left(\zeta_{o}, \zeta\right)
\end{aligned}
$$

with

$$
\Delta K\left(\zeta_{o}, \zeta\right)=\frac{z\left(\zeta_{o}\right) z(\zeta)}{j \beta a} \frac{w_{a}(\zeta) f_{a}\left(\zeta_{o}, \zeta\right)}{\phi_{a}{ }^{\prime}\left(\zeta_{o}, \zeta\right)} \frac{d z}{d \zeta} e^{-j \beta a \phi_{a}\left(\zeta_{o}, \zeta\right)}
$$

The full expression of $z(\zeta)$ and $\frac{d z}{d \zeta}$ is shown in Appendix C. The first term of $K\left(\zeta_{o}, \zeta\right)$ can be expressed in terms of a sinc function depending on the difference $\left(\zeta_{o}-\zeta\right)$.

In order to achieve this aim, first let us approximate the 


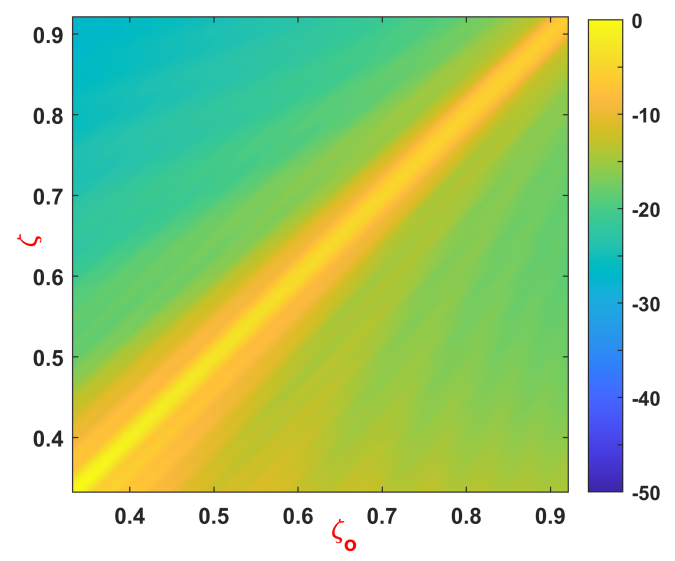

Fig. 6. Kernel of $\mathcal{T} \mathcal{T}^{\dagger}$ in the variables $\left(\zeta_{o}, \zeta\right)$ for $a=20 \lambda, x_{o}=10 \lambda$, $z_{\min }=2.5 \lambda,\left(\zeta_{\max }=0.920\right), z_{\max }=40 \lambda\left(\zeta_{\min }=0.333\right)$.

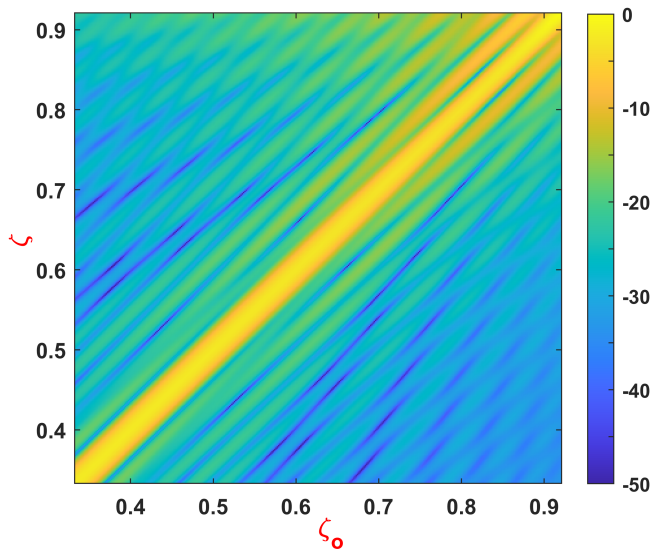

Fig. 7. Kernel of $\mathcal{T} \mathcal{T}_{w}^{\dagger}$ in the variables $\left(\zeta_{o}, \zeta\right)$ for $a=20 \lambda, x_{o}=10 \lambda$, $z_{\min }=2.5 \lambda\left(\zeta_{\max }=0.920\right), z_{\max }=40 \lambda\left(\zeta_{\min }=0.333\right)$.

amplitude term $z\left(\zeta_{o}\right) f_{-a}\left(\zeta_{o}, \zeta\right) / \phi_{-a}{ }^{\prime}\left(\zeta_{o}, \zeta\right)$ as in (15), and $f_{x_{o}}\left(\zeta_{o}, \zeta\right) / \sqrt{\phi_{x_{o}}^{\prime \prime}\left(\zeta_{o}, \zeta\right)}$ as below

$$
\frac{f_{x_{o}}\left(\zeta_{o}, \zeta\right)}{\sqrt{\left|\phi_{x_{o}}^{\prime \prime}\left(\zeta_{o}, \zeta\right)\right|}} \approx \frac{\sqrt{a}}{2 z(\zeta) z\left(\zeta_{o}\right)} \frac{\operatorname{sign}\left(\zeta_{o}-\zeta\right)}{\sqrt{\left|\frac{d z}{d \zeta}\right|}\left(\zeta_{o}-\zeta\right)}
$$

(see Appendix D for the derivation of approximation (26)). Later, let us choose the following weight function

$$
w\left(x^{\prime}, \zeta\right)=\frac{\bar{w}\left(x^{\prime}\right)}{z(\zeta)} \approx \frac{\frac{\bar{w}_{-a}-\bar{w}_{x_{o}}}{-a-x_{o}}\left(x^{\prime}-x_{o}\right)+\bar{w}_{x_{o}}}{z(\zeta)}
$$

where

$$
\bar{w}_{-a}=\frac{\pi\left(a+x_{o}\right)}{\lambda} \quad, \quad \bar{w}_{x_{o}}=\sqrt{\frac{a+x_{o}}{2 \lambda}} .
$$

By taking into account of equations (15) (26) (28), and by performing the following approximation

$$
\frac{1}{z(\zeta)} \sqrt{\left|\frac{d z}{d \zeta}\right|}=\frac{\sqrt{2}}{\sqrt{a+x_{o}}} \frac{\sqrt{1+\zeta^{2}}}{1-\zeta^{2}} \approx \sqrt{\frac{2}{a+x_{o}}}
$$

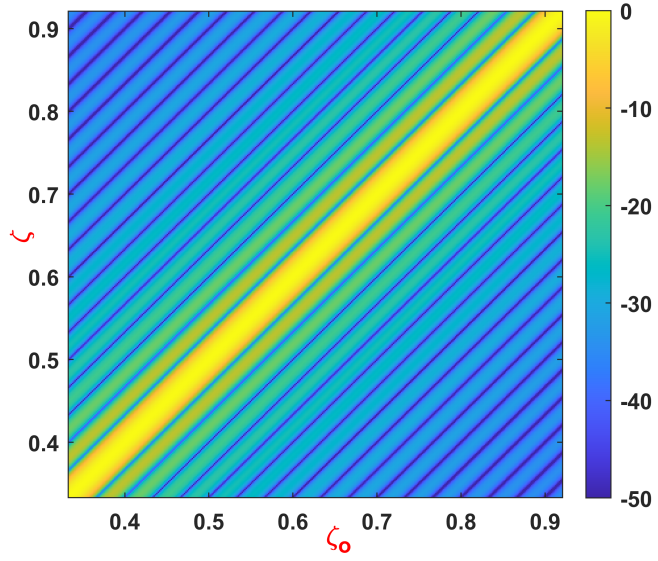

Fig. 8. Sinc kernel corresponding to the first term of (31) for $a=20 \lambda$, $x_{o}=10 \lambda, z_{\min }=2.5 \lambda\left(\zeta_{\max }=0.920\right), z_{\max }=40 \lambda\left(\zeta_{\min }=0.333\right)$.

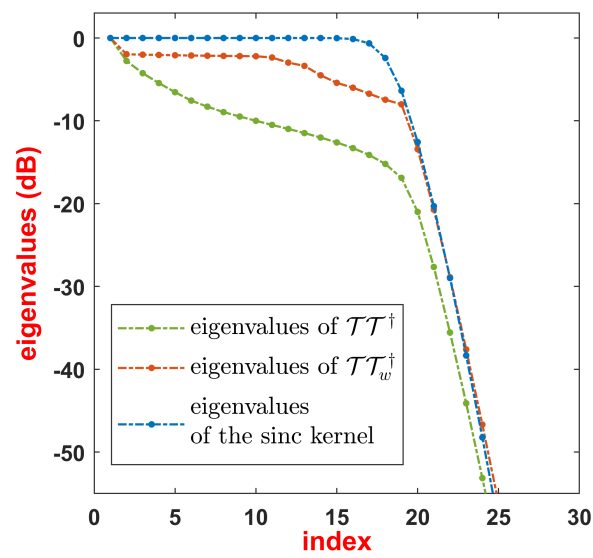

Fig. 9. Eigenvalues of $\mathcal{T} \mathcal{T}^{\dagger}, \mathcal{T} \mathcal{T}_{w}^{\dagger}$, and of the sinc kernel for $a=20 \lambda$, $x_{o}=10 \lambda, z_{\min }=2.5 \lambda\left(\zeta_{\max }=0.920\right), z_{\max }=40 \lambda\left(\zeta_{\min }=0.333\right)$.

the kernel expressed in (24) can be approximated as follows

$$
\begin{aligned}
& K\left(\zeta_{o}, \zeta\right) \approx \pi e^{-j \frac{\beta\left(a+x_{o}\right)}{2}\left(\gamma\left(\zeta_{o}\right)-\gamma(\zeta)\right)} e^{j \frac{\pi}{8} \operatorname{sign}\left(\zeta_{o}-\zeta\right)} \\
& \frac{\sin \left(\frac{\beta\left(a+x_{o}\right)}{2}\left(\zeta_{o}-\zeta\right)+\frac{\pi}{8} \operatorname{sign}\left(\zeta_{o}-\zeta\right)\right)}{\pi\left(\zeta_{o}-\zeta\right)}+\Delta K\left(\zeta_{o}, \zeta\right)
\end{aligned}
$$

Since equation (18) does not work for $z_{o}=z$, equation (30) is not valid for $\zeta_{o}=\zeta$. An expression of $K\left(\zeta_{o}, \zeta\right)$ which well approximates the behavior of the actual kernel $\forall\left(\zeta_{o}, \zeta\right) \in$ $\left[\zeta_{\min }, \zeta_{\max }\right] \times\left[\zeta_{\min }, \zeta_{\max }\right]$ is given by

$$
\begin{aligned}
K\left(\zeta_{o}, \zeta\right) \approx & \pi e^{-j \frac{\beta\left(a+x_{o}\right)}{2}\left(\gamma\left(\zeta_{o}\right)-\gamma(\zeta)\right)} \frac{\sin \left(\frac{\beta\left(a+x_{o}\right)}{2}\left(\zeta_{o}-\zeta\right)\right)}{\pi\left(\zeta_{o}-\zeta\right)} \\
& +\Delta K\left(\zeta_{o}, \zeta\right)
\end{aligned}
$$

Hence, if $0<x_{o}<a$ the kernel of $\mathcal{T} \mathcal{T}_{w}^{\dagger}$ is made up by two terms. The first one is proportional to a sinc kernel of difference type, instead, the second term $\Delta K$ can be regarded as an additive error.

Figures 6, 7 and 8 show respectively the actual kernel of $\mathcal{T T}^{\dagger}$, $\mathcal{T} \mathcal{T}_{w}^{\dagger}$, and the first term of (31). As it can be seen from such figures, the kernel of $\mathcal{T T}^{\dagger}$ exhibits a different decay with 


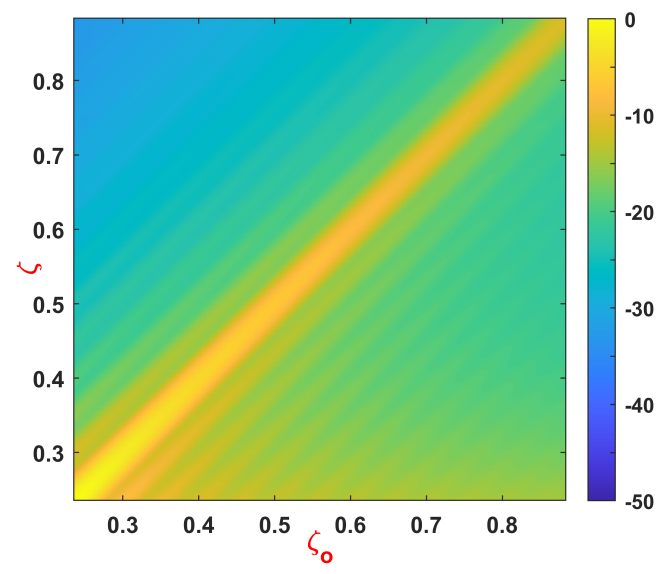

Fig. 10. Kernel of $\mathcal{T T}^{\dagger}$ in the variables $\left(\zeta_{o}, \zeta\right)$ for $a=20 \lambda, x_{o}=0 \lambda$, $z_{\min }=2.5 \lambda\left(\zeta_{\max }=0.883\right), z_{\max }=40 \lambda\left(\zeta_{\min }=0.236\right)$.

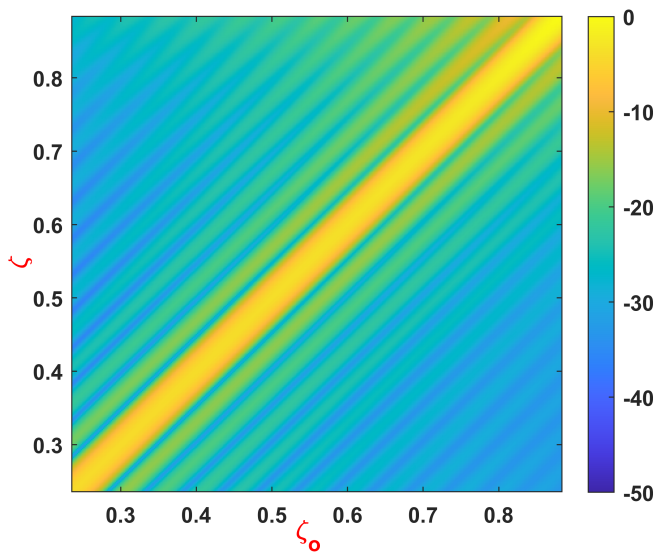

Fig. 11. Kernel of $\mathcal{T} \mathcal{T}_{w}^{\dagger}$ in the variables $\left(\zeta_{o}, \zeta\right)$ for $a=20 \lambda, x_{o}=0 \lambda$, $z_{\min }=2.5 \lambda\left(\zeta_{\max }=0.883\right), z_{\max }=40 \lambda\left(\zeta_{\min }=0.236\right)$.

respect to the others. Instead, the kernel of $\mathcal{T} \mathcal{T}_{w}^{\dagger}$ and the sinc term of (31) strongly differ in the region outside the main lobe whereas they are similar in the region of the main lobe (in particular way for small and medium values of $\zeta$ and $\zeta_{o}$ ). In this last case, the differences between the two diagrams are due essentially to the approximation made in (29), and to the fact that we are neglecting the additive term $\Delta K$.

Despite this, the number of significant eigenvalues is essentially the same in all the three cases. This aspect is well shown in fig. 9 which sketches the eigenvalues of $\mathcal{T} \mathcal{T}^{\dagger}, \mathcal{T} \mathcal{T}_{w}^{\dagger}$ and those of the integral operator with the sinc kernel. As it can be seen from the figure, the dynamics of the eigenvalues changes in each case, instead, the number of relevant eigenvalues is always the same. Hence, also for $0<x_{o}<a$ the number of relevant eigenvalues of $\mathcal{T} \mathcal{T}^{\dagger}$ can be computed by referring to integral operator with the sinc kernel corresponding to the first term of (31).

In the first part of this section, we have addressed the cases where $0<x_{o}<a$; instead, now we are going to tackle the case where $x_{o}=0$ and $x_{o}=a$. As we will see, in such cases the asymptotic evaluation of the kernel can be expressed only by two terms and this leads to a kernel whose expression is

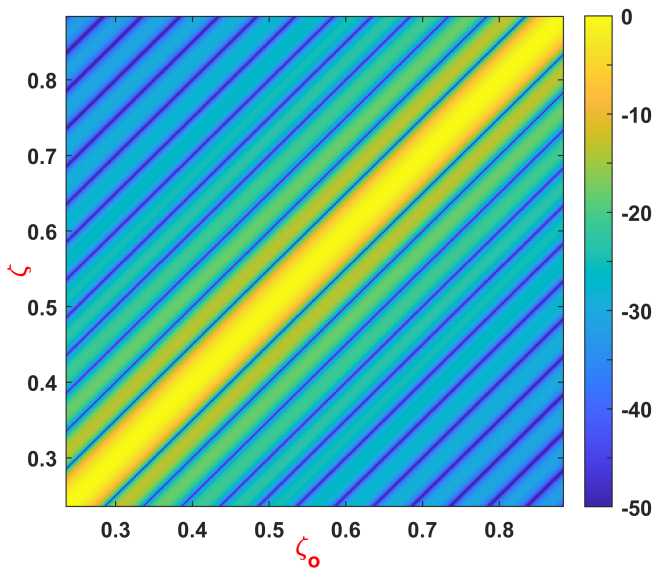

Fig. 12. Sinc kernel given by equation (35) for $a=20 \lambda, x_{o}=0 \lambda$, $z_{\min }=2.5 \lambda\left(\zeta_{\max }=0.883\right), z_{\max }=40 \lambda\left(\zeta_{\min }=0.236\right)$.

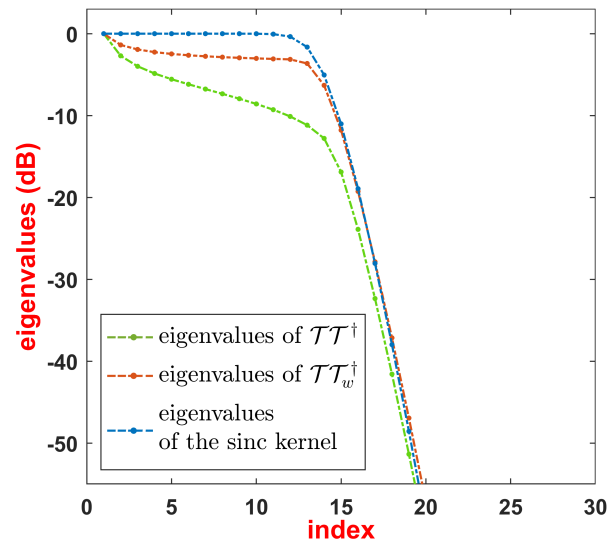

Fig. 13. Eigenvalues of $\mathcal{T} \mathcal{T}^{\dagger}, \mathcal{T} \mathcal{T}_{w}^{\dagger}$, and of the sinc kernel for $a=20 \lambda$, $x_{o}=0 \lambda, z_{\min }=2.5 \lambda\left(\zeta_{\max }=0.883\right), z_{\max }=40 \lambda\left(\zeta_{\min }=0.236\right)$.

given only by the first term of (31).

\section{A. The case $x_{o}=0$}

In this section we will study the operator $\mathcal{T} \mathcal{T}_{w}^{\dagger}$ in the case where $x_{0}=0$. In such case, the asymptotic evaluation of $K\left(z_{o}, z\right)$ provided by equation (18) still works $\forall\left(z_{o}, z\right) \in$ $\left[z_{\text {min }}, z_{\text {max }}\right] \times\left[z_{\text {min }}, z_{\text {max }}\right]:\left\{z_{o} \neq z\right\}$. Furthermore, since $x_{o}=0$, it results also that

$$
\phi_{a}\left(z_{o}, z\right)=\phi_{-a}\left(z_{o}, z\right) \quad, \quad \frac{f_{a}\left(z_{o}, z\right)}{\phi_{a}^{\prime}\left(z_{o}, z\right)}=-\frac{f_{-a}\left(z_{o}, z\right)}{\phi_{-a}^{\prime}\left(z_{o}, z\right)}
$$

Consequently, if the weight function $w\left(x^{\prime}, z\right)$ is such that $w_{a}(z)=w_{-a}(z)$, the kernel (18) can be rewritten as follows

$$
\begin{aligned}
& K\left(z_{o}, z\right) \approx z_{o} z\left(\frac{2 w_{-a} f_{-a}}{j \beta a \phi_{-a}^{\prime}} e^{-j \beta a \phi_{-a}}+\right. \\
&\left.+\sqrt{\frac{2 \pi}{\beta a}} \frac{w_{x_{o}} f_{x_{o}}}{\sqrt{\left|\phi_{x_{o}}^{\prime \prime}\right|}} e^{-j \beta a \phi_{x_{o}}} e^{j \frac{\pi}{4} \operatorname{sign}\left(-\phi_{x_{o}}^{\prime \prime}\right)}\right)
\end{aligned}
$$

where the subscript 0 denotes the the correspondent function has been particularized for $x^{\prime}=0$. After the introduction of the variables $\zeta$ and $\gamma$ (see equations (21) and (22)), if we 


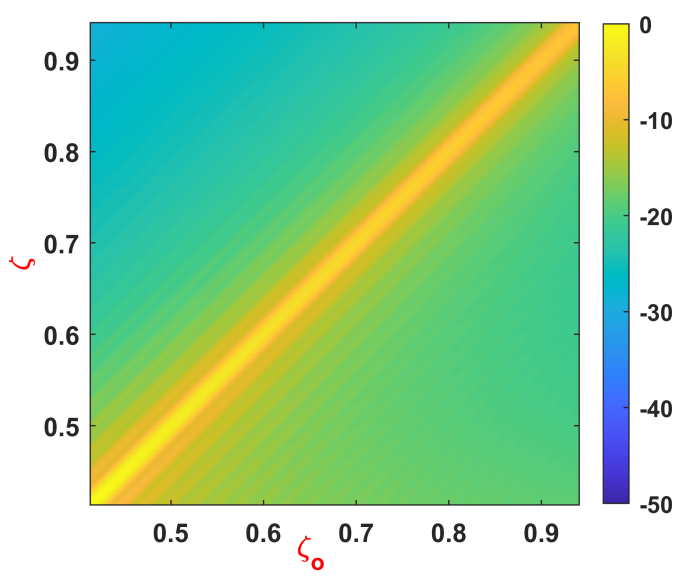

Fig. 14. Kernel of $\mathcal{T} \mathcal{T}^{\dagger}$ in the variables $\left(\zeta_{o}, \zeta\right)$ for $a=20 \lambda, x_{o}=20 \lambda$, $z_{\min }=2.5 \lambda\left(\zeta_{\max }=0.939\right), z_{\max }=40 \lambda\left(\zeta_{\min }=0.415\right)$.

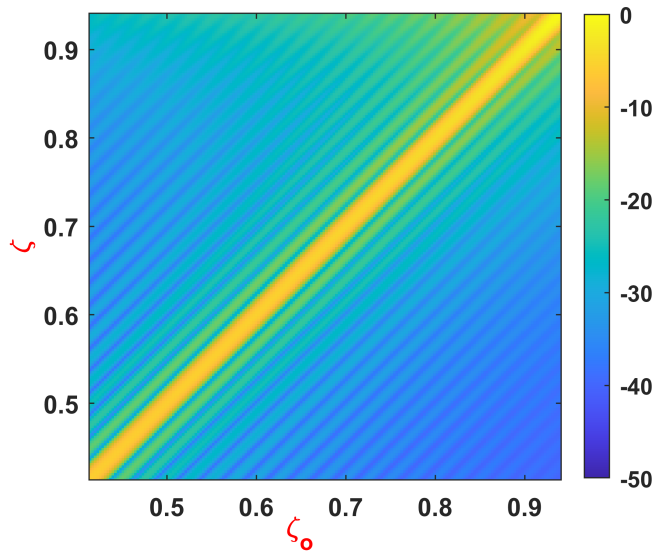

Fig. 15. Kernel of $\mathcal{T} \mathcal{T}_{w}^{\dagger}$ in the variables $\left(\zeta_{o}, \zeta\right)$ for $a=20 \lambda, x_{o}=20 \lambda$, $z_{\min }=2.5 \lambda\left(\zeta_{\max }=0.939\right), z_{\max }=40 \lambda\left(\zeta_{\min }=0.415\right)$.

repeat the same discussions made for the case $0<x_{o}<a$ and if we choose as weight function

$$
w\left(x^{\prime}, \zeta\right)=\frac{\bar{w}\left(x^{\prime}\right)}{z(\zeta)}=\frac{\frac{\bar{w}_{-a}-\bar{w}_{0}}{-a}\left|x^{\prime}\right|+\bar{w}_{0}}{z(\zeta)}
$$

with $\bar{w}_{-a}=\frac{\pi a}{\lambda}$ and $\bar{w}_{0}=\sqrt{\frac{2 a}{\lambda}}$, we obtain that the kernel of $\mathcal{T} \mathcal{T}_{w}^{\dagger}$ can be recast as below

$$
K\left(\zeta_{o}, \zeta\right) \approx 2 \pi e^{-j \frac{\beta a}{2}\left(\gamma\left(\zeta_{o}\right)-\gamma(\zeta)\right)} \frac{\sin \left(\frac{\beta a}{2}\left(\zeta_{o}-\zeta\right)\right)}{\pi\left(\zeta_{o}-\zeta\right)} .
$$

In figures 10,11 and 12 the kernel of $\mathcal{T} \mathcal{T}^{\dagger}, \mathcal{T} \mathcal{T}_{w}^{\dagger}$, and the sinc kernel (35) are sketched in $d B$. Note that if $x_{o}=0$, the sinc kernel expressed by (35) approximates well the actual kernel of $\mathcal{T} \mathcal{T}_{w}^{\dagger}$. The only difference between the two diagrams concerns the value of the two kernels along the lines given by the equation $\zeta_{o}-\zeta=n \frac{2 \pi}{\beta a}$. Along such lines, the sinc kernel is exactly zero, instead, the actual kernel of $\mathcal{T} \mathcal{T}_{w}^{\dagger}$ assume a value that is $-35 d B$ with respect to its maximum.

In fig. 13 the eigenvalues of $\mathcal{T} \mathcal{T}^{\dagger}, \mathcal{T} \mathcal{T}_{w}^{\dagger}$ and those of the integral operator with the sinc kernel (35) are depicted. As it

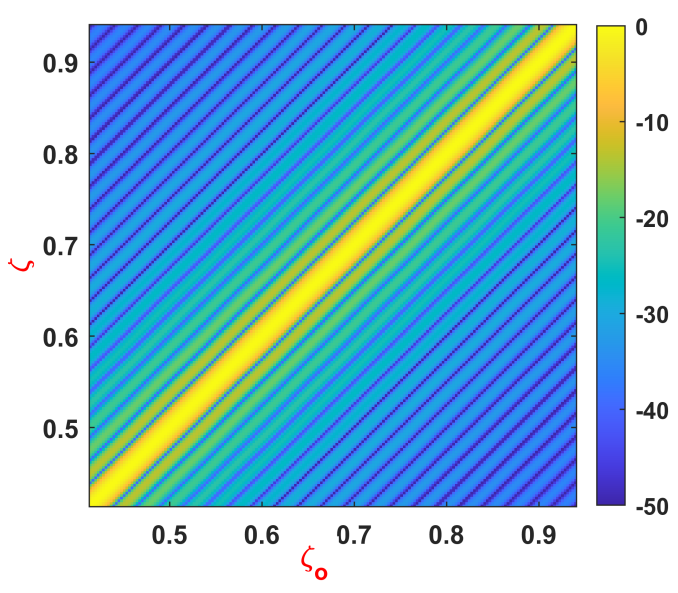

Fig. 16. Sinc kernel given by equation (35) for $a=20 \lambda, x_{o}=20 \lambda$, $z_{\min }=2.5 \lambda\left(\zeta_{\max }=0.939\right), z_{\max }=40 \lambda\left(\zeta_{\min }=0.415\right)$.

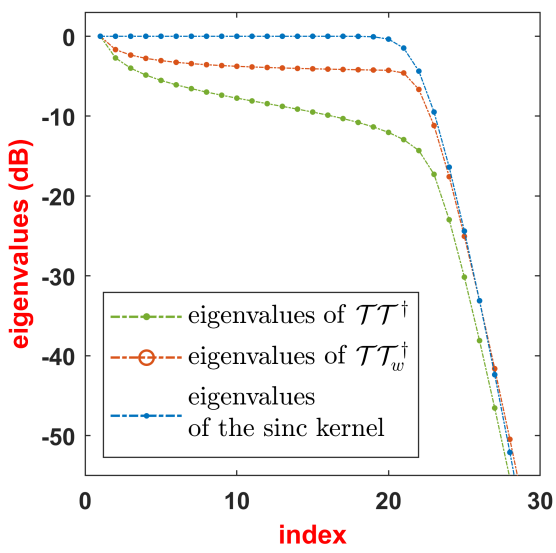

Fig. 17. Eigenvalues of $\mathcal{T} \mathcal{T}^{\dagger}, \mathcal{T} \mathcal{T}_{w}^{\dagger}$, and of the sinc kernel for $a=20 \lambda$, $x_{o}=20 \lambda, z_{\min }=2.5 \lambda\left(\zeta_{\max }=0.939\right), z_{\max }=40 \lambda\left(\zeta_{\min }=0.415\right)$.

can be seen from the figure, also for $x_{o}=0$ the number of relevant eigenvalues is the same in all the cases. Consequently, also for $x_{o}=0$ the number of relevant eigenvalues of $\mathcal{T}^{\dagger}$ can be computed by referring to integral operator with the sinc kernel (35).

\section{B. The case $x_{o}=a$}

In this section, we will study the operator $\mathcal{T} \mathcal{T}_{w}^{\dagger}$ in the case $x_{o}=a$. In such a case, the endpoint $x^{\prime}=a$ is also a stationary point, hence, the asymptotic evaluation of the kernel $K\left(z_{o}, z\right)$ is given by

$$
\begin{aligned}
K\left(z_{o}, z\right) \approx z_{o} z & \left(\frac{w_{-a} f_{-a}}{j \beta a \phi_{-a}} e^{-j \beta a \phi_{-a}}+\right. \\
+ & \left.\frac{1}{2} \sqrt{\frac{2 \pi}{\beta a}} \frac{w_{a} f_{a}}{\sqrt{\left|\phi_{a}^{\prime \prime}\right|}} e^{-j \beta a \phi_{a}} e^{j \frac{\pi}{4} \operatorname{sign}\left(-\phi_{a}^{\prime \prime}\right)}\right)
\end{aligned}
$$

After the introduction of the variables $\zeta$ and $\gamma$ defined in (21) and (22), if we repeat the same reasoning made for the case $0<x_{o}<a$ and if we choose the weight function as follows

$$
w\left(x^{\prime}, \zeta\right)=\frac{\bar{w}\left(x^{\prime}\right)}{z(\zeta)}=\frac{\frac{\bar{w}_{-a}-\bar{w}_{a}}{-a-a} x^{\prime}+\bar{w}_{a}}{z(\zeta)}
$$


with $\bar{w}_{-a}=\frac{2 \pi a}{\lambda}$ and $\bar{w}_{a}=\sqrt{\frac{4 a}{\lambda}}$, we obtain that the kernel of $\mathcal{T} \mathcal{T}_{w}^{\dagger}$ can be recast as below

$$
K\left(\zeta_{o}, \zeta\right) \approx \pi e^{-j \beta a\left(\gamma\left(\zeta_{o}\right)-\gamma(\zeta)\right)} \frac{\sin \left(\beta a\left(\zeta_{o}-\zeta\right)\right)}{\pi\left(\zeta_{o}-\zeta\right)} .
$$

In figures 14,15 and 16 the kernel of $\mathcal{T} \mathcal{T}^{\dagger}, \mathcal{T} \mathcal{T}_{w}^{\dagger}$, and the sinc kernel are sketched in $d B$. Instead, in figure 17 the eigenvalues of $\mathcal{T} \mathcal{T}^{\dagger}, \mathcal{T} \mathcal{T}_{w}^{\dagger}$ and those of the sinc kernel (38). As can be seen from the figure, in the case $x_{o}=a$ all the consideration already made for $x_{o}=0$ hold true.

\section{NDF OF THE RADIATED FIELD}

In the previous sections, we have shown that the eigenvalues of $\mathcal{T T}^{\dagger}$ can exhibit a different dynamics from those of the correspondent integral operator with the sinc kernel but in each case the position of the knee in the eigenvalues diagrams occurs at the same index. For such reason, the number of degrees of freedom (that corresponds to the number of eigenvalues before the knee) can be evaluated by referring to the integral operator below

$$
\int_{\hat{\zeta}\left(z_{\max }\right)}^{\hat{\zeta}\left(z_{\min }\right)} e^{j W\left(\hat{\gamma}(\zeta)-\hat{\gamma}\left(\zeta_{o}\right)\right)} \frac{\sin \left(W\left(\hat{\zeta}_{o}-\hat{\zeta}\right)\right)}{\pi\left(\hat{\zeta}_{o}-\hat{\zeta}\right)}(.) d \zeta
$$

where

$$
W= \begin{cases}\beta \frac{a+x_{o}}{2} & \text { for } 0 \leq x_{o} \leq a \\ \beta a & \text { for } x_{o}>a\end{cases}
$$

By exploiting the Slepian-Pollak results in [30], we obtain that the number of degrees of freedom are given by

$$
N D F=\frac{W}{\pi}\left(\hat{\zeta}\left(z_{\min }\right)-\hat{\zeta}\left(z_{\max }\right)\right)
$$

where $\hat{\zeta}(z)$ has the behavior shown in fig. 18 and it is given by

$\hat{\zeta}(z)=\left\{\begin{array}{l}\frac{\sqrt{\left(x_{o}+a\right)^{2}+z^{2}}-|z|}{a+x_{o}} \text { for } 0 \leq x_{o} \leq a \\ \frac{\sqrt{\left(x_{o}+a\right)^{2}+z^{2}}-\sqrt{\left(x_{o}-a\right)^{2}+z^{2}}}{2 a} \text { for } x_{o} \geq a\end{array}\right.$

As can be seen from equation (41), the NDF depends only on the configuration parameters which are $a, x_{o}, z_{\min }, z_{\max }$. Note that if $\zeta\left(z_{\min }\right)$ and $\zeta\left(z_{\max }\right)$ remain unchanged, and $x_{o}$ changes in the set $x_{o} \in[0, a]$ then the NDF increases with respect to $x_{o}$. Differently, if $\zeta\left(z_{\min }\right)$ and $\zeta\left(z_{\max }\right)$ remain unchanged, and $x_{o}$ changes in the set $x_{o} \in[a, \infty]$ then the NDF remains unchanged.

Let us remark that if the observation domain was infinite, the difference $\hat{\zeta}\left(z_{\min }\right)-\hat{\zeta}\left(z_{\max }\right)$ would be equal to 1 , and consequently, the NDF would be at maximum $(\beta a) / \pi$. Since in practical cases the observation domain is necessarily finite, the NDF are always less than $(\beta a) / \pi$.

Bearing in mind that the set of all possible currents is well represented with $(2 \beta a) / \pi$ independent functions, it follows that it is not possible to retrieve the current if the data are collected only on 1 line orthogonal to the source.

In order to retrieve the current, the data may be collected

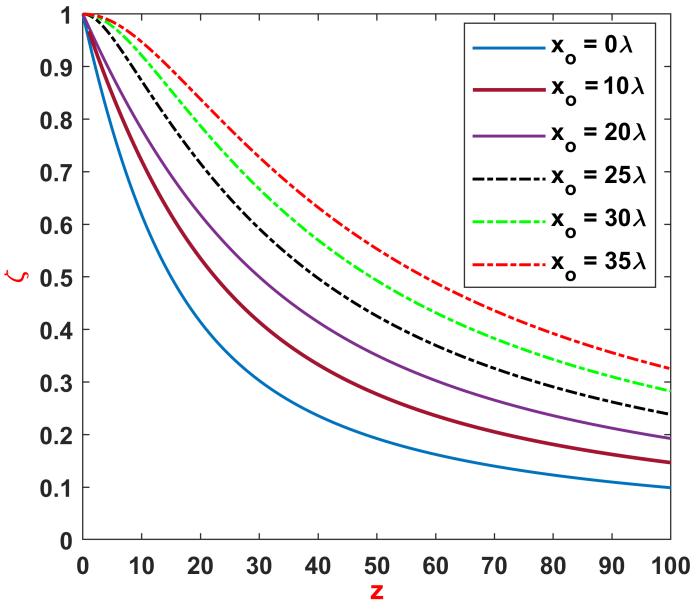

Fig. 18. Behavior of the transformation $\zeta(z)$ for different values of $x_{o}$ when $a=20 \lambda$.

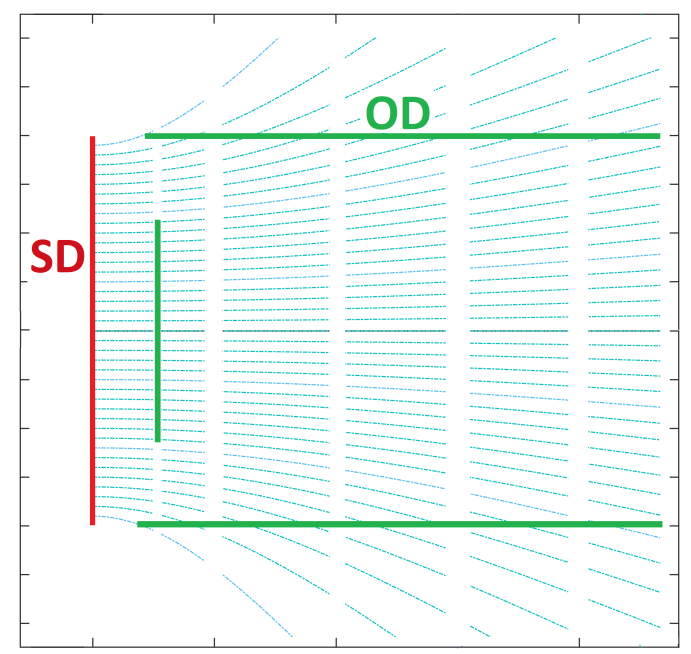

Fig. 19. Geometry with combined observation domain.

on 2 scanning lines of infinite extension placed along the axis $x_{o}=a$ and $x_{o}=-a$. However, since the observation domains are necessarily limited, we must add also a little observation domain parallel to the source. The described configuration is shown in fig. 19 and it can be completely understood by linking the result provided here and those shown in [34].

\section{CONCLUSION}

In this paper we have provide an analytical expression of the NDF of the radiated field with reference to a magnetic strip current observed on a truncated line parallel to the source. Our result works also in near zone, hence it extends the results of the Fresnel zone shown in [28] and the results of the near zone for a centered configuration shown [29].

It is worth noting that this study, and that developed in [34] represent the mathematical basis for addressing configurations where the observation domain is a generic curve. The latter are very interesting in applications since a generic curve of observation may represent the trajectory followed by an UAV- 
based system for in-situ evaluation of radiating systems [35][39]. More in detail, the analysis made in [34] is suitable to study the case where the observation domain is a smooth curve slowly varying (in such case no stationary points appear in the kernel of the integral equation involved in the computation of the singular values). Instead, the analysis that we develop in this article allows to study configurations where the observation domain is a smooth curve rapidly varying (in such case a stationary point appears in the kernel of the integral equation for the computation of the singular values).

\section{APPENDIX A}

THE TRANSFORMATION, ITS INVERSE, AND THE DERIVATIVE OF THE INVERSE FOR THE CASE $x_{o}>a$.

In this appendix, with reference to the the transformation

$$
\zeta(z)=\frac{1}{2 a}\left(\sqrt{\left(x_{o}+a\right)^{2}+z^{2}}-\sqrt{\left(x_{o}-a\right)^{2}+z^{2}}\right)
$$

used for $x_{o}>a$, we provide the expression of $z(\zeta)$ and $\frac{d z}{d \zeta}$. In particular, the inverse function $z(\zeta)$ is given by

$$
z(\zeta)=\sqrt{\left(1-\zeta^{2}\right)\left(\frac{x_{o}^{2}}{\zeta^{2}}-a^{2}\right)}
$$

while its derivative is provided by

$$
\frac{d z}{d \zeta}=\frac{a^{2} \zeta-x_{o}^{2} \zeta^{-3}}{z(\zeta)}
$$

\section{APPENDIX B}

\section{APPROXIMATION OF THE AMPLITUDE TERMS}

$$
\frac{z\left(\zeta_{o}\right) f_{a}\left(\zeta_{o}, \zeta\right)}{\phi_{a}^{\prime}\left(\zeta_{o}\right), \zeta} \text { AND } \frac{z\left(\zeta_{o}\right) f_{-a}\left(\zeta, \zeta_{o}\right)}{\phi_{-a}^{\prime}\left(\zeta, \zeta_{o}\right)}
$$

In this appendix, we show how to derive the approximation of the amplitude terms $z\left(\zeta_{o}\right) \frac{f_{a}\left(\zeta_{o}, \zeta\right)}{\phi_{a}^{\prime}\left(\zeta_{o}, \zeta\right)}$ and $z\left(\zeta_{o}\right) \frac{f_{-a}\left(\zeta_{o}, \zeta\right)}{\phi_{-a}^{\prime}\left(\zeta_{o}, \zeta\right)}$ made in (14) and (15).

In order to obtain equation (14), we have performed the following approximation

$$
\frac{z_{o} f_{a}\left(z_{o}, z\right)}{\phi_{a}^{\prime}\left(z_{o}, z\right)} \approx \frac{\left.z_{o} f_{a}\left(z_{o}, z\right)\right|_{z_{o}=z}}{\left.\phi_{a}^{\prime}\left(z_{o}, z\right)\right|_{z_{o}=z}+\left.\frac{d \phi_{a}^{\prime}\left(z_{o}, z\right)}{d z_{o}}\right|_{z_{o}=z}\left(z_{o}-z\right)}
$$

Taking into account for the definitions of $f\left(x^{\prime}, z_{o}, z\right)$ and $\phi\left(x^{\prime}, z_{o}, z\right)$ provided in section III, it results that

$$
\begin{gathered}
\left.z_{o} f_{a}\left(z_{o}, z\right)\right|_{z_{o}=z}=\frac{z}{\left[\left(x_{o}-a\right)^{2}+z^{2}\right]^{3 / 2}} \\
\left.\phi_{a}^{\prime}\left(z_{o}, z\right)\right|_{z_{o}=z}=0 \\
\left.\frac{d \phi_{a}^{\prime}\left(z_{o}, z\right)}{d z_{o}}\right|_{z_{o}=z}=\frac{z\left(x_{o}-a\right)}{a\left[\left(x_{o}-a\right)^{2}+z^{2}\right]^{3 / 2}}
\end{gathered}
$$

Consequently, equation (44) can be rewritten as below

$$
\frac{z_{o} f_{a}\left(z_{o}, z\right)}{\phi_{a}^{\prime}\left(z_{o}, z\right)} \approx \frac{a}{\left(x_{o}-a\right)\left(z_{o}-z\right)}
$$

By exploiting the equation $z_{o}-z=\frac{d z}{d \zeta}\left(\zeta_{o}-\zeta\right)$, it results that

$$
\frac{z\left(\zeta_{o}\right) f_{a}\left(\zeta_{o}, \zeta\right)}{\phi_{a}^{\prime}\left(\zeta_{o}, \zeta\right)} \approx \frac{a}{\left(x_{o}-a\right)\left(\zeta_{o}-\zeta\right) \frac{d z}{d \zeta}}
$$

The same discussion has been repeated also to approximate the term $z\left(\zeta_{o}\right) \frac{f_{-a}\left(\zeta_{o}, \zeta\right)}{\phi_{-a}^{\prime}\left(\zeta_{o}, \zeta\right)}$.

\section{APPENDIX C}

THE TRANSFORMATION, ITS INVERSE, AND THE DERIVATIVE OF THE INVERSE FOR THE CASE $0<x_{o}<a$. In this appendix, with reference to the the transformation

$$
\zeta(z)=\frac{1}{a+x_{o}}\left(\sqrt{\left(x_{o}+a\right)^{2}+z^{2}}-|z|\right)
$$

used for $0<x_{o}<a$, we provide the expression of $z(\zeta)$ and $\frac{d z}{d \zeta}$. In particular, the inverse function $z(\zeta)$ has the following expression

$$
z(\zeta)=\frac{a+x_{o}}{2}\left(\frac{1}{\zeta}-\zeta\right)
$$

while its derivative is given by

$$
\frac{d z}{d \zeta}=-\frac{a+x_{o}}{2}\left(\frac{1}{\zeta^{2}}+1\right) .
$$

\section{APPENDIX D
APPROXIMATION OF THE TERM $\frac{f_{x_{o}}\left(\zeta_{o}, \zeta\right)}{\sqrt{\left|\phi_{x_{o}}^{\prime \prime}\left(\zeta_{o}, \zeta\right)\right|}}$}

In this appendix, we justify the approximation (26).

Taking into account for the definitions of $f\left(x^{\prime}, z_{o}, z\right)$ and $\phi\left(x^{\prime}, z_{o}, z\right)$ provided in section III, it results that

$$
\frac{f_{x_{o}}\left(z_{o}, z\right)}{\sqrt{\left|\phi_{x_{o}}^{\prime \prime}\left(z_{o}, z\right)\right|}}=\frac{1}{z z_{o}} \frac{\sqrt{a}}{\sqrt{\left|z_{o}-z\right|}}
$$

Since $z_{o}-z=\frac{d z}{d \zeta}\left(\zeta_{o}-\zeta\right)$, equation (52) can be rewritten as below

$$
\frac{f_{x_{o}}\left(\zeta_{o}, \zeta\right)}{\sqrt{\left|\phi_{x_{o}}^{\prime \prime}\left(\zeta_{o}, \zeta\right)\right|}}=\frac{1}{2 z(\zeta) z\left(\zeta_{o}\right)} \frac{2 \sqrt{a}}{\sqrt{\left|\frac{d z}{d \zeta}\right|} \sqrt{\left|\zeta_{o}-\zeta\right|}}
$$

By performing the following approximation

$$
\frac{1}{\zeta_{o}-\zeta} \approx \frac{2}{\sqrt{\left|\zeta_{o}-\zeta\right|}} \operatorname{sign}\left(\zeta_{o}-\zeta\right)
$$

we obtain that

$$
\frac{f_{x_{o}}\left(\zeta_{o}, \zeta\right)}{\sqrt{\left|\phi_{x_{o}}^{\prime \prime}\left(\zeta_{o}, \zeta\right)\right|}} \approx \frac{\sqrt{a}}{2 z(\zeta) z\left(\zeta_{o}\right)} \frac{\operatorname{sign}\left(\zeta_{o}-\zeta\right)}{\sqrt{\left|\frac{d z}{d \zeta}\right|}\left(\zeta_{o}-\zeta\right)}
$$

\section{REFERENCES}

[1] G. Schnattinger, and T. Eibert, "Solution to the full vectorial 3D inverse source problem by multilevel fast multipole method inspired hierarchical disaggregation," IEEE Trans. Antennas Propag., vol. 60, no. 7, pp. 3325$3335,2012$.

[2] L. J. Foged, L. Scialacqua, F. Saccardi, J. L. A. Quijano, G. Vecchi, and M. Sabbadini, "Practical Application of the Equivalent Source Method as an Antenna Diagnostics Tool," IEEE Antennas Propag. Mag., vol. 54, no. 5, pp. 243-249, Oct. 2012.

[3] E.A. Marengo, and A.J. Devaney, "The inverse source problem of electromagnetics: Linear inversion formulation and minimum energy solution," IEEE Trans. Antennas Propag., vol. 47, pp. 410-412, 1999.

[4] C. Cappellin, A. Frandsen, and O. Breinbjerg, "Application of the SWEto-PWE Antenna Diagnostics Technique to an Offset Reflector Antenna", IEEE Trans. Antennas Propag. Mag., vol. 50, no. 5, pp. 204-213, Oct. 2008 
[5] R.A.M. Mauermayer, Y. Weitsch, and T.F. Eibert, "Electromagnetic Field Synthesis by Hierarchical Plane Wave-Based Field Transformation," IEEE Trans. Antennas Propag., vol. 63, pp. 5561-5572, 2015.

[6] J. L. Araque Quijano, and G. Vecchi, "Improved-Accuracy Source Reconstruction on Arbitrary 3D Surfaces," IEEE Antennas Wirel. Propag. Lett., vol. 8, pp. 1046-1049, 2009.

[7] Y. Alvarez, F. Las-Heras, B. A. Dominguez-Casas, and C. Garcia, "Antenna Diagnostics Using Arbitrary-Geometry Field Acquisition Domains," IEEE Antennas Wirel. Propag. Lett., vol. 8, pp. 375-378, 2009.

[8] J. Kornprobst, R.A.M. Mauermayer, O. Neitz, J. Knapp, and T. Eibert, "On the Solution of Inverse Equivalent Surface-Source Problems" Prog. Electromagn. Res., vol. 165, pp. 4765, 2019.

[9] K. Persson, M. Gustafsson and G. Kristensson, "Reconstruction and Visualization of Equivalent Currents on a Radome Using an Integral Representation Formulation", Progress in Electromagnetics Research B, vol. 20, pp. 65-90, 2010.

[10] A.J. den Dekker, and A. van den Bos, "Resolution: a survey," J. Opt. Soc. Am. A, vol. 14 , no. 3, pp. 547557, 1997.

[11] G.T Di Francia, "Degrees of freedom of an image," J. Opt. Soc. Am. A, vol. 59, no. 7, pp. 799-804, 1969.

[12] R. Piestun, and D.A.B. Miller, "Electromagnetic degrees of freedom of an optical system," J. Opt. Soc. Am. A, vol. 17, pp. 892-902, 2000.

[13] B. Fuchs, and A. G. Polimeridis, "Reduced Order Models for Fast Antenna Characterization," IEEE Trans. Antennas Propag., vol. 67, no. 8, pp. 5673-5677, Aug. 2019.

[14] K. Xu, L. Ran, Y. Zhong, and X. Chen, "Singular value decomposition of the current-to-field operator in solving inverse scattering problems," 2014 IEEE Antennas and Propagation Society International Symposium (APS-URSI), Memphis, TN, 2014, pp. 659-660.

[15] David G. Fischer, Richard A. Frazin, Marius Asipauskas, and P. Scott Carney, "Information content of the near field: three-dimensional samples," J. Opt. Soc. Am. A, vol. 28, pp. 296-306, 2011

[16] T. Yin, Z. Wei and X. Chen, "Non-Iterative Methods Based on Singular Value Decomposition for Inverse Scattering Problems," IEEE Trans. on Antennas Propag., vol. 68, no. 6, pp. 4764-4773, Jun. 2020.

[17] G. Gennarelli, I. Catapano, F. Soldovieri and R. Persico, "On the Achievable Imaging Performance in Full 3-D Linear Inverse Scattering," IEEE Trans. on Antennas Propag., vol. 63, no. 3, pp. 1150-1155, March 2015.

[18] T. Isernia, G. Leone, R. Pierri "Sulle dimensioni essenziali dei campi: un approccio ai valori singolari," IX Riunione Nazionale di Elettromagnetismo, Assisi, Itay, 1992

[19] R. Somaraju, and J. Trumpf, "Degrees of freedom of a communication channel: using DOF singular values," IEEE Trans. Inf. Theory, vol. 56, pp. 1560-1573, 2010.

[20] R. Pierri, and F. Soldovieri, "On the information content of the radiated fields in the near zone over bounded domains," Inverse Problems, vol. 14, no. 2, pp. 321-337, 1998.

[21] M. A. Maisto, R. Solimene, and R. Pierri, "Resolution limits in inverse source problem for strip currents not in Fresnel zone," J. Opt. Soc. Am. A, vol. 36, pp. 826-833, 2019.

[22] G. Leone, F. Munno and R. Pierri, "Inverse Source on Conformal Conic Geometries," IEEE Trans. Antennas Propag., 2020.

[23] A. S. Y. Poon, R. W. Brodersen, and D. N. C. Tse, "Degrees of freedom in multiple-antenna channels: a signal space approach," IEEE Trans. Inf. Theory, vol. 51, no. 2, pp. 523-536, Feb. 2005.

[24] A. Capozzoli, C. Curcio, A. Liseno, "Multi-frequency planar near-field scanning by means of singular-value decomposition (SVD) optimization, IEEE Antennas Prop. Mag., vol. 53, no. 6, pp. 212-221, Dec. 2011.

[25] R. Solimene, M.A. Maisto, R. Pierri, "Sampling approach for singular system computation of a radiation operator," J. Opt. Soc. Am. A, vol. 36, no. 3, pp. 353-361, 2019.

[26] J. Ranieri, A. Chebira, and M. Vetterli, "Near-Optimal Sensor Placement for Linear Inverse Problems," IEEE Trans. Signal Process., vol. 62, no. 5, pp. 1135-1146, March 2014.

[27] C. Jiang, Y. Soh, and H. Li "Sensor placement by maximal projection on minimum eigenspace for linear inverse problems," IEEE Trans. Signal Process., vol. 64, pp. 5595-5610, 2016.

[28] R. Pierri, A. Liseno, F. Soldovieri, and R. Solimene, "In-depth resolution for a strip source in the Fresnel zone," J. Opt. Soc. Am. A, vol. 18, no. 2, pp. 352-359, 2001.

[29] M.A. Maisto, R. Solimene, and R. Pierri, "Depth resolution in strip current reconstructions in near non-reactive zone," J. Opt. Soc. Am. A, vol. 36, no. 6, pp. 975-982, 2019.

[30] D. Slepian, and H.O. Pollack, "Prolate spheroidal wave functions, Fourier analysis, and uncertainty-I, The Bell System Technical Journal, vol. 40, no. 1, pp. 43, 1961.
[31] K. Khare, and N. George, "Sampling theory approach to prolate spheroidal wavefunctions," Journal of Physics A: Mathematical and General, vol. 36, no. 39, pp. 10011, 2003.

[32] M. Bertero, and P. Boccacci. Introduction to Inverse Problems in Imaging; IOP Publishing: Bristol, UK, 1998.

[33] N. Bleistein, R.A. Handelsman. Asymptotic Expansions of Integrals; Dover Publications: New York, NY, USA, 1986.

[34] R. Pierri, and R. Moretta, "Asymptotic study of the radiation operator for the strip current in near zone" Electronics, vol. 9, no. 6, art. no. 911, 2020.

[35] G. Virone et al., "Antenna pattern verification system based on a micro unmanned aerial vehicle (UAV)", IEEE Antennas Wireless Propag. Lett., vol. 13, pp. 169-172, 2014.

[36] T. Fritzel, R. Strau, H.J. Steiner, C. Eisner, and T. Eibert, "Introduction into an UAV-based near-field system for in-situ and large-scale antenna measurements," IEEE Conference on Antenna Measurements \& Applications (CAMA), pp. 1-3, 2016.

[37] M. G. Fernandez et al. "Antenna Diagnostics and Characterization using Unmanned Aerial Vehicles," IEEE Access, vol. 5, pp. 23563-23575, 2017.

[38] G. Virone et al. "Strong Mutual Coupling Effects on LOFAR: Modeling and In Situ Validation," IEEE Trans. Antennas Propag., vol. 66, no. 5, pp. $2581-2588,2018$

[39] L. Ciorba et al. "Near-Field Phase Reconstruction for UAV-based Antenna Measurements," 13th European Conference on Antennas and Propagation (EuCAP), 2019.

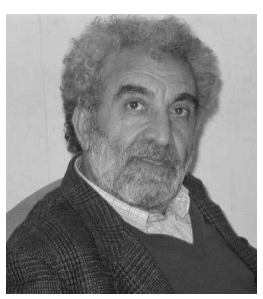

Rocco Pierri received the Laurea degree (summa cum laude) in electronic engineering from the University of Naples "Federico II" in 1976.

He was with the university of Naples "Federico II", Naples. He was a Visiting Scholar with the University of Illinois at Urbana-Champaign, Urbana, IL, USA; Harvard University, Cambridge, MA, USA; Northeastern University, Boston, MA, USA; Supelec, Paris, France; and the University of Leeds, Leeds, U.K. He also extensively lectured abroad in many universities and research centers.

$\mathrm{He}$ is currently a Full Professor with the University of Campania "Luigi Vanvitelli", Aversa, Italy. His current research interests include antennas, phase retrieval, near-field techniques, inverse electromagnetic scattering, subsurface sensing, electromagnetic diagnostics, microwave tomography, inverse source problems, and information content of radiated field.

Prof. Pierri was a recipient of the 1999 Honorable Mention for the $\mathrm{H}$. A. Wheeler Applications Prize Paper Award of the IEEE Antennas and Propagation Society.

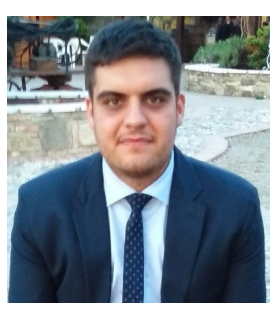

Raffaele Moretta received M.S. degree (summa cum laude) in electronic engineering from the University of Campania "Luigi Vanvitelli" in 2018, where he is currently pursuing the Ph.D. Degree.

Since 2016, he has been started a scientific cooperation with the Electromagnetic Fields Group of the University of Campania "Luigi Vanvitelli". His current research interests include inverse problems in electromagnetics with particular attention to phase retrieval, and near field techniques. 